\title{
Practical Strategies for cryo-CLEM Experiments
}

\author{
Cheri M. Hampton ${ }^{1}$
}

1. Department of Pediatrics, Division of Infectious Diseases, Emory University School of Medicine, Atlanta, GA

As our ability to discern higher resolution structure of biological macromolecules increases, the need to interpret these structures in the context of the living cell becomes apparent. This tutorial will highlight the use of cryo-CLEM[1] to link physiological information from cell biology with structural studies of cells, microorganisms and macromolecules using the best of multiple imaging modalities. Much of the strategy involves selection of ideal cells for culture and vitrification as well as appropriate labelling options. Once the system is optimized, the techniques include, but are not limited to cryo-fluorescence microscopy; cryoelectron microscopy; and cryo-electron tomography. Applications include cellular architecture; protein expression and localization; host-pathogen interactions and virus structure[2].

The first of many strategy considerations is the choice of cultured cell line to use for cryo-CLEM experiments. The electron beam is only able to penetrate regions of the cell up to 500nm, so while the cell nucleus may be off limits, events in the thinner regions of the cell are amenable to in situ imaging. Choosing cell lines that are adherent and spreading will go a long way towards being able to visualize the cellular event. Addition of extracellular adhesion factors such as collagen or fibronectin can improve adhesion for some cell types. Likewise, cell density on the TEM grid support film will have an effect on the ability to plunge freeze the cells instead of the more involved high pressure freezing options.

Other initial strategies include choice of labeling to visualize the cellular event. Options vary from whole cell labeling to fluorescently-tagged protein expression, or infection/transfection with more sophisticated labeled protein components.

Once frozen, grids are then imaged by cryo-fluorescence microscopy. This method is preferable to live cell imaging prior to freezing in that (1) the cells and fluorescent labels do not move between imaging modes, and (2) in many cases the cryo-fluorescent signal is resistant to quenching[3]. For time-sensitive events, series of grids frozen at specific time points can be beneficial.

There are several models of cryo-stages commercially available. The principle objective is to obtain image maps and coordinates of labeled events on the grid that are transferrable to the coordinate system of the TEM imaging software. Several options exist, and new ones are being developed[4,5]. Use of additional extracellular fiduciary markers can facilitate registration of maps as well as improve resolvability of fluorescent targets[6,7].

The specific applications of this technique are broad and range from localization of cells of interest, localization of specifically tagged proteins within a cell, virus fusion/interaction, to engineered nanoprobe targeting of proteins or nucleic acids.

References:

[1] JM Plitzko, A Rigort, A Leis. Current Opinion in Biotechnology 20 (2009), p. 83. 
[2] CM Hampton, et al, Nature Protocols 12 (2017), p. 150.

[3] CL Schwartz, et al. Journal of Microscopy 227 (2007), p. 98.

[4] W Kukulski, et al, Journal of Cell Biology 192 (2011), p. 111.

[5] M Schorb and JAG Briggs. Ultramicroscopy 143 (2014), p. 24.

[6] P Schellenberger, et al,Ultramicroscopy 143 (2014), p. 41.

[7] M Schorb, et al. Journal of Structural Biology 197 (2017), p. 83.

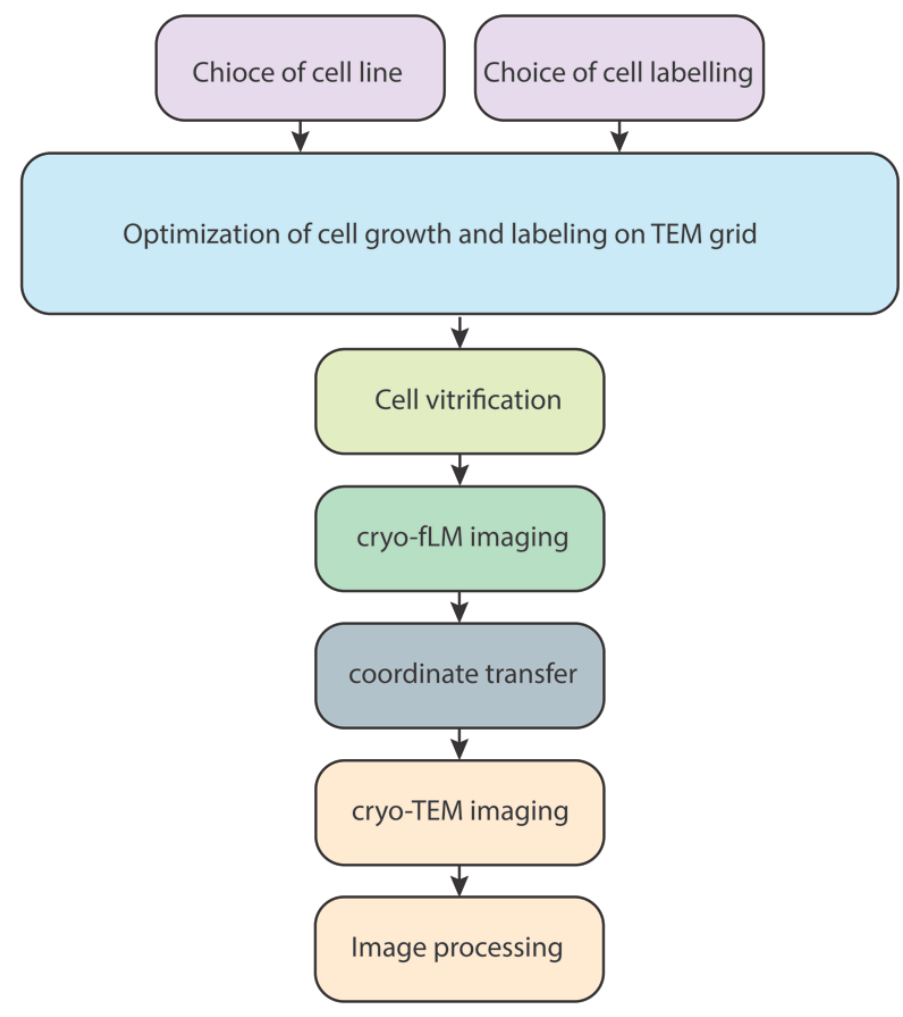

Figure 1. Simplified flowchart of cryo-CLEM imaging. Initial steps are weighted more heavily in terms of contribution to successful outcomes. 